\title{
Radiation Environment on the International Space Station During the Solar Particle Events in March 2012
}

\author{
Jordanka Semkova ${ }^{1 *}$, Tsvetan Dachev¹, Rositza Koleva1, Stefan Maltchev, Nikolay Bankov", Victor Benghin², Vyacheslav Shurshakov², \\ Vladislav Petrov ${ }^{2}$ and Sergey Drobyshev ${ }^{2}$
}

${ }^{1}$ Space research and technology institute, Bulgarian academy of sciences, Bulgaria

${ }^{2}$ State scientific centre of Russian federation, institute of biomedical problems, Russian academy of sciences, Russia

\begin{abstract}
The Liulin-5 charged particle telescope observes the radiation characteristics in the spherical tissue-equivalent phantom of MATROSHKA-R international project on the International Space Station (ISS) since June 2007. In this paper attention is drawn to the results from the measurements of dose rate and particle flux increase during the Solar Energetic Particles Events (SPE) occurred in March 2012. During that SPE the solar particles penetrated at high geographic latitudes in the regions of the south and north Earth magnetic poles and at $3<L$ they caused particle flux and dose rates increase in all three detectors of Liulin-5, located at 40,60 and $165 \mathrm{~mm}$ depths along the phantom's radius. The maximum flux at $40 \mathrm{~mm}$ depth observed outside the South Atlantic Anomaly (SAA) during that SPE reached 7.2 part $/ \mathrm{cm}^{2}$.s and the dose rate reached $107.8 \mu \mathrm{Gy} / \mathrm{h}$ on 07.03.2012, 13:06 UT at L=4. The additional absorbed dose received from SPE is approximately $180 \mu \mathrm{Gy}$ and additional dose equivalent- approximately $448 \mu \mathrm{Sv}$. These additional exposures are comparable to the averaged daily absorbed dose and dose equivalent measured in the spherical phantom in ISS during quite radiation conditions. Discussed are the linear energy transfer spectra measured and quality factors obtained during and after the SPE. Compared are data from Liulin-5 and other particle detectors in space during the SPE.
\end{abstract}

Keywords: Space radiation dosimetry; International space station; Solar particles event

\section{Introduction}

Space radiation is a very important component of the space weather and affects both the space crew and the electronic devices in space flights. The radiation field in the ISS is complex, composed of Galactic Cosmic Rays (GCR), trapped radiation of the Earth radiation belts, solar energetic particles, albedo particles from Earth's atmosphere and the secondary radiation produced in the shielding materials of the spacecraft and in human body.

The GCRs, consisting of $99 \%$ protons and He nuclei and $1 \%$ heavy ions with energies up to tens of $\mathrm{GeV} /$ nuc are a permanent source of ionizing radiation in the ISS. The GCR radiation in the near - Earth free space is approximately isotropic. $\mathrm{Up}$ to $1 \mathrm{GeV}$ the flux and spectra of GCR particles are strongly influenced by the solar activity and hence show modulation that is anti-correlated with solar activity.

The biological impact of space radiation to humans depends strongly on the particle's Linear Energy Transfer (LET) and is dominated by high LET radiation. Especially important is the effect of the high energy heavy ion component of GCR, possessing high LET and highly penetrating in human body, which provides them with a large potential for radiobiological damage.

Another component of the incident radiation field in the ISS orbit is the trapped protons and electrons. The trapped protons of the inner radiation belt have energies up to several hundreds of $\mathrm{MeV}$ and contribute a large fraction of the dose rates outside and inside the ISS. The trapped protons are encountered by LEO spacecraft in the region of the South Atlantic Anomaly (SAA). Although only about $5 \%$ of the mission time of the ISS is spent in the SAA, the astronauts may collect more than $50 \%$ of their total absorbed dose during this short time period [1,2].

The average kinetic energy of the inner zone trapped electrons is a few hundred $\mathrm{keV}$. These electrons are easily removed from the spacecraft interior by the slightest amount of shielding and are mainly of concern to astronauts during extra-vehicular activities. At higher latitudes the ISS crosses the earthward part of the outer electron radiation belt. The average energy of these electrons is also about few hundred $\mathrm{keV}$.

Solar Particle Events (short-term high-intensity bursts of protons and ions accelerated to hundreds of $\mathrm{MeV}$ ) also contribute transient increases to the radiation environment. There are two ways SPE can influence the radiation situation on low Earth orbit. The first one is the well known direct penetration of solar energetic particles into the magnetosphere. Only particles with energies higher than the geomagnetic cut-off energy can penetrate into the space station orbit. The time scale of such phenomena is about one-two days $[3,4]$. The second one is an increase of the trapped particle flux when SPE particles act as an additional source of particles for trapping and subsequent radial difusion into the inner magnetosphere. At some geomagnetic conditions SPE energetic particles can penetrate in the inner belt region and be trapped there $[5,6]$. In such case the SPE-origin energetic particles can change dramatically the radiation environment on low earth orbit on a long-term time scale (up to several months). The Liulin radiometer-dosimeter onboard Mir space station [7] has collected data about radiation environment during number of SPE in SeptemberOctober 1989, March 1991, June 1991 and June 1992 [8-11]. During the SPE on September 291989 when the space station reached high latitudes in the near-polar geomagnetic regions $(\mathrm{L}>3.5)$ dose rate and

${ }^{*}$ Corresponding author: Jordanka Semkova, Space research and technology institute, Bulgarian academy of sciences, Bulgaria, E-mail: jsemkova@stil.bas.bg

Received March 20, 2013; Accepted June 24, 2013; Published June 26, 2013

Citation: Semkova J, Dachev T, Koleva R, Maltchev S, Bankov N, et al. (2013) Radiation Environment on the International Space Station During the Solar Particle Events in March 2012. Astrobiol Outreach 1: 102. doi: 10.4172/2332-2519.1000102

Copyright: (c) 2013 Semkova J, et al. This is an open-access article distributed under the terms of the Creative Commons Attribution License, which permits unrestricted use, distribution, and reproduction in any medium, provided the original author and source are credited. 
Citation: Semkova J, Dachev T, Koleva R, Maltchev S, Bankov N, et al. (2013) Radiation Environment on the International Space Station During the Solar Particle Events in March 2012. Astrobiol Outreach 1: 102. doi: 10.4172/2332-2519.1000102

flux show a sharp rise up to (8-9)x10-2 Gy/day and 200-250 cm-2 s-1, respectively. The flux exceeded 200-250 times and the dose rate exceeded 500-600 times the common for those regions values in the absence of SPE.

Dose characteristics in LEO depend also on many other parameters such as the solar cycle phase; spacecraft orbit parameters, helio - and geophysical parameters.

For the estimation of the organ doses from the complex radiation field in the ISS, and thus the radiation risk, measurements in human phantoms are essential. In 2004 the MATROSHKA-R international experiment was started on the Russian segment of the ISS. The experiment MATROSHKA-R includes the Russian spherical tissueequivalent phantom [12], equipped with passive and active experiment packages for studies of the depth dose distribution at various sides of the organs of a human body exposed to cosmic radiation. Liulin-5 is an active experiment in the spherical phantom. The aim of the Liulin-5 experiment is a long-term investigation of the radiation quantities in the phantom, using a telescope of three silicon detectors. The first stage of the Liulin-5 experiment on the ISS took place from June 2007 to June 2010, corresponding to very quiet solar conditions during the deep minimum of the $23 \mathrm{rd}$ solar cycle. Some results from those investigations can be found in [2,13-15]. The second stage of the Liulin-5 experiment is conducted on the ISS since December 2011 to obtain data for radiation conditions during the solar activity increase in the 24th cycle. From December 2011 to May 2012 the spherical phantom with Liulin-5 instrument was located in the MIM1 module of the Russian Segment of the ISS. In this paper we present results of Liulin-5 measurements of the radiation parameters during the SPE, occurred in the time interval 7-12.03.2012.

\section{Liulin-5 Method and Instrument}

The Liulin-5 particle telescope is mounted inside the largest diameter channel of the phantom Figure 1. More detailed description of Liulin-5 method and instrument can be found in [13]. The detector module of Liulin-5 contains three silicon detectors D1, D2 and D3 arranged as a telescope. The detectors axis is along the phantom's

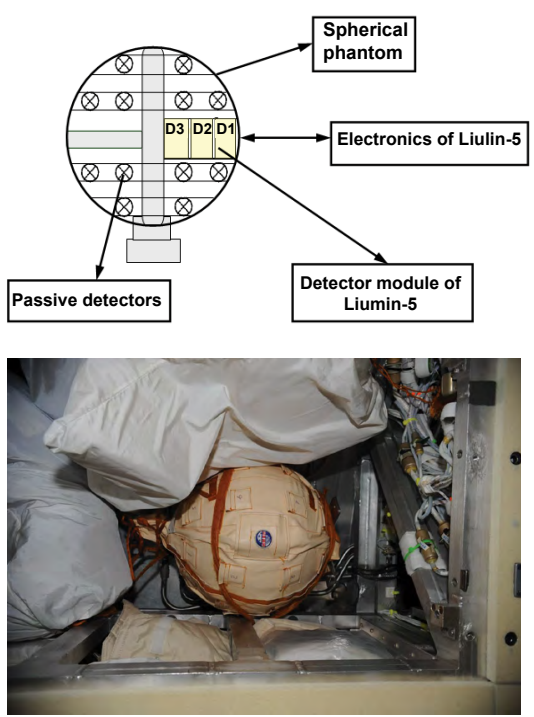

Figure 1: Liulin-5 onboard ISS. Upper- sketch of the detectors arrangement in the phantom, bottom- the spherical phantom, located in the MIM1 module. Inside the phantom (behind the label) is the detector module of Liulin-5. radius. The D1 detector is at $40 \mathrm{~mm}, \mathrm{D} 2$ is at $60 \mathrm{~mm}$ and D3 is at 165 $\mathrm{mm}$ distance from the phantom's surface.

The position of D1 and D2 in the phantom corresponds approximately to the depth of the blood forming organs in human body, while D3 is placed very close to the phantom's centre. This arrangement allows measuring the dose-depth distribution along the sphere's radius.

The investigation of the radiation environment in the phantom in ISS by the Liulin- 5 experiment envisages: i) measurement of the depth distributions of the energy deposition spectra, flux and dose rate, and absorbed dose D; ii) measurement of the LET spectrum in silicon, and then calculation of LET spectrum in water and Q, according to the Q (L) relationship given in ICRP60 [16], where L stays for LET. Q (L) is related functionally to the unrestricted LET of a given radiation, and is multiplied by the absorbed dose to derive the dose equivalent $\mathrm{H}$.

\section{Results and Discussions}

The results presented here deal with the flux and dose rates, LET spectra, obtained quality factors $\mathrm{Q}$ and dose equivalent values during and after SPE on March 07-12, 2012.

\section{Distribution of dose rates and particle fluxes during quite conditions}

A typical distribution of Liulin-5 particle flux and dose rate as a function of L-value is presented in Figure 2. The data represent the measurements in detector D1 about 11 hours after the end of SPE observed on ISS orbit (see below). Well seen are the two main sources of radiation in a low Earth orbit - the GCR and the trapped protons of the inner radiation belt in SAA. At L values 1.1-1.8 both sources contribute to the measured flux. Maximum fluxes of $19.3 \mathrm{part} / \mathrm{cm}^{2} \mathrm{~s}$ are registered from the trapped protons in SAA at $\mathrm{L} \sim 1.24$. Minima values of about $0.035 \mathrm{part} / \mathrm{cm}^{2}$ s were recorded at $\mathrm{L} \sim 1$ from GCR. The maximum dose rate in SAA was $370 \mu \mathrm{Gy} / \mathrm{h}$. Outside SAA the averaged flux was 0.2 part $/ \mathrm{cm}^{2}$.s., averaged absorbed dose rate $2.65 \mu \mathrm{Gy} / \mathrm{h}$.

\section{Distribution of dose rates and particle fluxes along the ISS orbits during SPE}

On 07.03.2012 GOES -13 registered the beginning of two SEP events (Proton fluxes at $\geq 10 \mathrm{MeV}$ exceeding the threshold of 10 part/ $\mathrm{cm}^{2}$.s.sr.) at geostationary orbit. The greater than $100 \mathrm{MeV}$ event began at $04: 05 \mathrm{Z}$ on 07.03 .2012 , reached a maximum of 69 part/.s.sr. at $15: 25 \mathrm{Z}$ the same day, and ended at $16: 50 \mathrm{Z}$ on 10.03.2012. Also, a greater than $10 \mathrm{MeV}$ event began at $05: 10 \mathrm{Z}$ on 07.03 .2012 , reached a

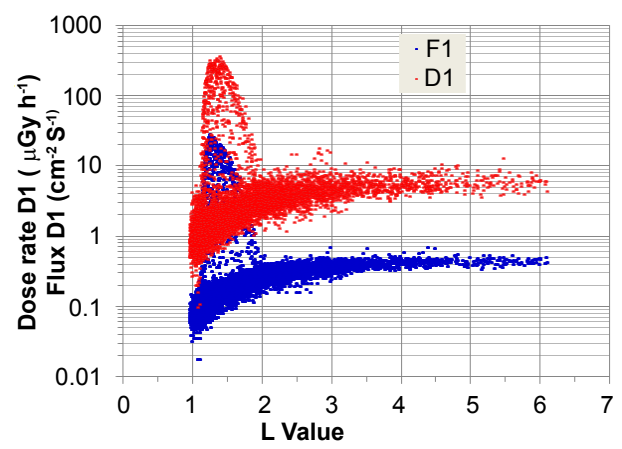

Figure 2: Distribution of particle flux $F 1$ (blue) and dose rate (red) in D1 detector located at $40 \mathrm{~mm}$ depth in the phantom as a function of $L$ value during quite conditions. 
Citation: Semkova J, Dachev T, Koleva R, Maltchev S, Bankov N, et al. (2013) Radiation Environment on the International Space Station During the Solar Particle Events in March 2012. Astrobiol Outreach 1: 102. doi: 10.4172/2332-2519.1000102

maximum of 6530 part $/ \mathrm{cm}^{2}$.s.sr. at $11: 15 \mathrm{Z}$ on 08.03 .2012 and ended at 20:50 Z on 12.03.2012. The flares were associated with Earth-directed CMEs (ftp://ftp.ngdc.noaa.gov/STP/swpc_products/weekly_reports/ PRFs_of_SGD/2012/03/prf1906.pdf).

The first registration of particle flux and dose rate increase in Liulin-5 data was on 07.03.2012, at 13:01 UT, at $\mathrm{L}=3$, Lat $=-42.3^{0}$, Long $=136.6^{\circ}$, Alt $=421.7 \mathrm{~km}$. The last registration of flux and dose rate increase was on 08.03.2012, at 21:31 UT at $\mathrm{L}=3.8$, Lat $=-48.8^{0}$, Long $=71.3^{\circ}$, Alt $=421.7 \mathrm{~km}$. The increase of the particle flux and dose rates at $\mathrm{L}>3$ is observed in all three detectors of Liulin-5 located at 40, 60 and $165 \mathrm{~mm}$ depth along the radius of the spherical phantom. In Figure 3 the distribution of the particle flux and the dose rate in D1 detector of Liulin-5 as a function of L-value is presented for the time interval 07.03.2012, 12:59 UT to 08.03.2012, 21:31 UT. In Figure 4 the dose rate distribution in D1 for the same interval obtained outside SAA is presented in geographical coordinates. It is seen that Liulin-5 flux and dose rate increase is observed at $3<\mathrm{L}$ at high geographic latitudes in the regions of the south and north Earth magnetic poles.

The maximum flux observed outside SAA during that SPE reached $7.2 \mathrm{part} / \mathrm{cm}^{2} . \mathrm{s}$ and the dose rate reached $107.8 \mu \mathrm{Gy} / \mathrm{h}$ on 07.03 .2012 , 13:06 UT at L $=4$, Lat $=-51.1^{0}$, Long $=166.8^{0}$, Alt $=422 \mathrm{~km}$. The averaged absorbed dose rate D1 outside SAA during SPE was $8.43 \mu \mathrm{Gy} / \mathrm{h}$ and the averaged flux was $0.59 \mathrm{part} / \mathrm{cm}^{2}$.s. The total additional absorbed dose received from the solar energetic particles was about $180 \mu \mathrm{Gy}$.

In Figure 5 the particle flux $\mathrm{F}$ of protons with energies $\geq 100$ $\mathrm{MeV}$ measured by GOES-13 (http://www.swpc.noaa.gov/ftpdir/ warehouse/2012/2012_plots/proton/20120309_proton.gif), the dose rate in D1 detector of Liulin -5 measured outside SAA, and L-values are plotted versus time from March 7, 07:00 UT to March 9, 22:00 UT, 2012. It is seen that the trend of Liulin -5 dose rate during that period of the SPE corresponds to the trend of the proton flux with energies above $100 \mathrm{MeV}$ (protons with lower energies do not penetrate inside ISS). After March 9, 06:00 UT GOES $\geq 100 \mathrm{MeV}$ proton flux is less than

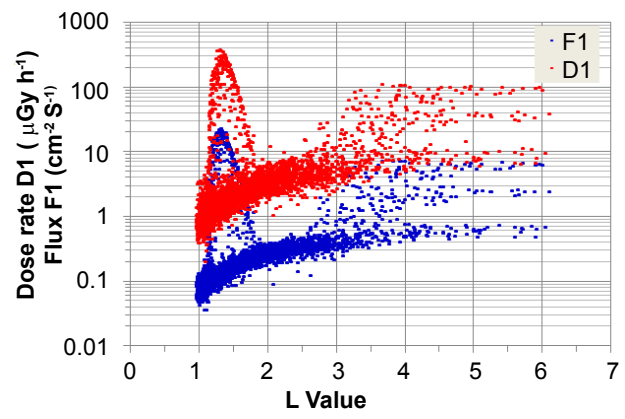

Figure 3: Distribution of particle flux $\mathrm{F} 1$ (blue) and dose rate (red) in D1 detector located at $40 \mathrm{~mm}$ depth in the phantom as a function of $L$ value during SPE.

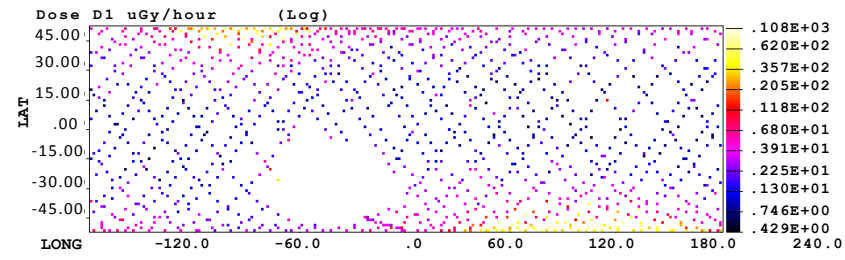

Figure 4: Geographical distribution of dose rate in detector D1 measured outside SAA during SPE.
10 part $/ \mathrm{cm}^{2} . s . s r$ and Liulin- 5 dose rate goes down to its almost normal values of $10-12 \mu \mathrm{Gy} / \mathrm{h}$ at $\mathrm{L}>3$.

\section{LET Spectra, quality factor and dose equivalent}

The LET spectra, obtained during and after the SPE taken on ISS orbits outside SAA are plotted in Figure 6 together with the corresponding orbits. The differences in both LET spectra are due to the difference in their sources - outside SAA the spectrum during SPE includes GCR (protons and heavier ions) and solar particles (protons mainly), the spectrum after the SPE is composed of GCR only. The quality factor $\mathrm{Q}_{\mathrm{av}}$ calculated from the LET spectrum during the SPE was 2.5 , leading to $21.1 \mu \mathrm{Sv} / \mathrm{h}$ dose equivalent rate at $40 \mathrm{~mm}$ depth. After the SPE $Q_{a v}$ was 4.15 , leading to $11 \mu \mathrm{Sv} / \mathrm{h}$ dose equivalent. Significant contribution to the GCR $\mathrm{Q}_{\mathrm{av}}$ has heavy ions (even though they are a small number of the total number of particles forming the GCR LET spectrum) with higher $\mathrm{Q}_{\mathrm{av}}$. During the SPE the LET spectrum outside the SAA is dominated by protons of solar origin, having smaller $\mathrm{Q}_{\mathrm{av}}$. The additional dose equivalent at $40 \mathrm{~mm}$ depth in the phantom received from SPE is approximately $448 \mu \mathrm{Sv}$, which is comparable to the daily dose equivalent at that depth during quite periods.

\section{Comparison between doses measured in Liulin 5 and DB-8 dosimeter on ISS}

In Figure 7 the accumulated doses in Liulin- 5 and in DB-8

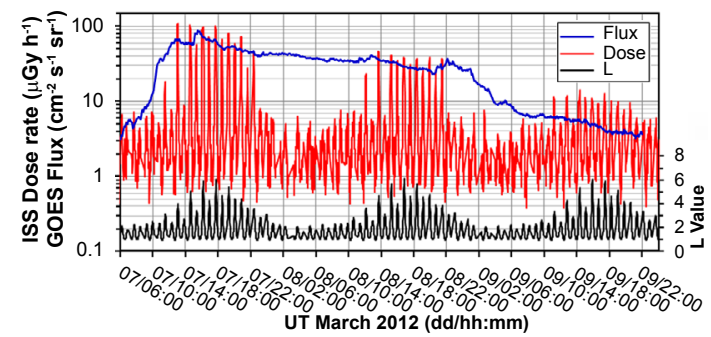

Figure 5: GOES-13 flux data of protons with energies above $100 \mathrm{MeV}$ (blue curve), Liulin-5 dose rate measured outside SAA (red curve) and the corresponding $L$ values (black curve) versus time during SPE.
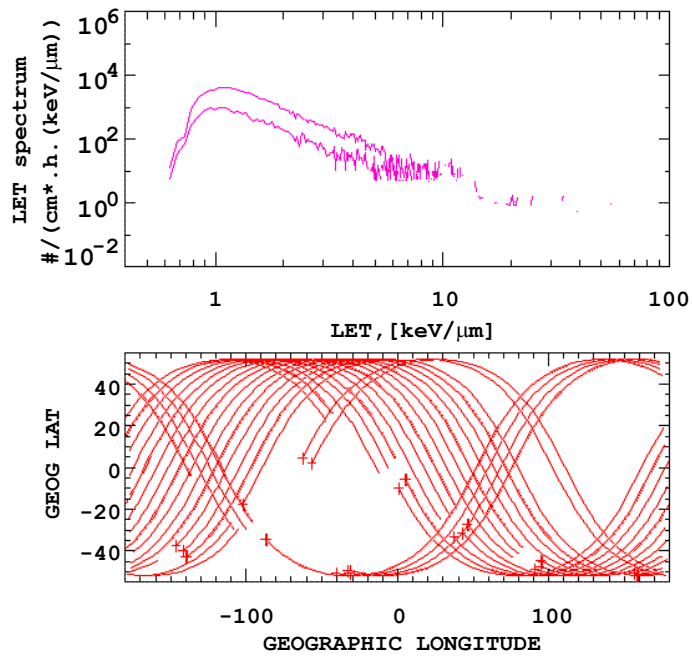

Figure 6: LET spectra (top plot) and the corresponding ISS orbits (bottom plot). Upper curve in the spectra - measurements during SPE, bottom curve measurements after the end of SPE. 


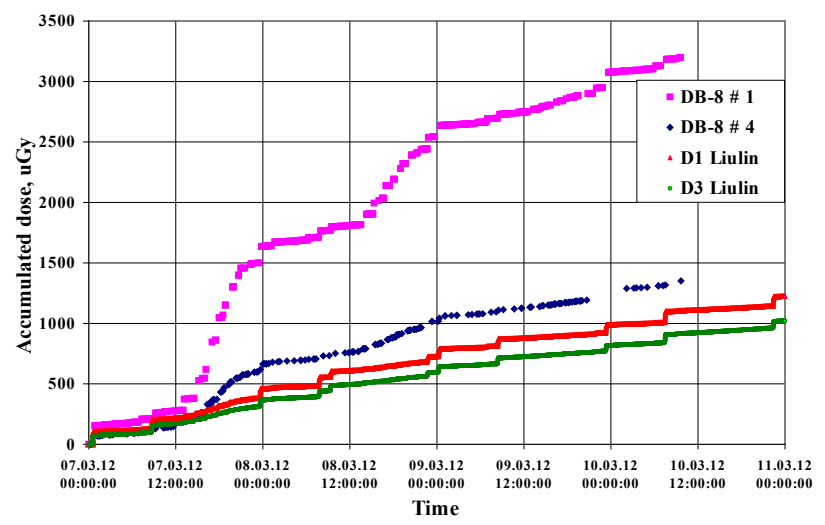

Figure 7: Comparison between accumulated doses measured in the detectors of Liulin -5 (two lower curves) and DB-8 dosimeters (two higher curves) in the time interval 07.03.2012, UT 00:00 to 11.03. 2012, UT 00:00.

active dosimeter of the radiation monitoring system on the Russian segment of the ISS [17] in the time interval 07.03.2012, 00:00 UT to 11.03. 2012, 00:00 UT are plotted. The top two curves represent the data in the non-shielded detectors DB-8\#1 and DB-8\#4, located in different positions in the service module of the ISS. Two bottom curves represent the accumulated doses in Liulin- 5 detectors D3 (innermost) and D1 (outermost) in the phantom, located in MIM1 module of the ISS. Well seen is the sharp increase of the accumulated doses in the data from DB-8 between 07.03.2012, 12:00UT and 09.03.2012, 00:00UT connected with the appearance of SPE. The accumulated in Liulin-5 detectors doses show similar but not so sharp trend, because they are heavier shielded inside the phantom.

\section{Conclusion}

During the SPE of 7-12 March 2012 at $3<\mathrm{L}$ the particle flux and dose rates increased in all three detectors of Liulin- 5 charged particle telescope located at 40,60 and $165 \mathrm{~mm}$ depths along the radius of the tissue-equivalent spherical phantom in MIM1 module of ISS. The maximum flux at $40 \mathrm{~mm}$ depth outside the SAA during the SPE was $7.2 \mathrm{part} / \mathrm{cm}^{2}$.s and the maximum dose rate was $107.8 \mu \mathrm{Gy} / \mathrm{h}$ at $\mathrm{L}=4$, Latitude $=-51.1^{0}$, Longitude $=166.8^{\circ}$, Altitude $=422 \mathrm{~km}$. The averaged dose rate outside the SAA during the SPE was $8.43 \mu \mathrm{Gy} / \mathrm{h}$ and the flux was $0.59 \mathrm{part} / \mathrm{cm}^{2} . \mathrm{s}$. The averaged dose rate outside the SAA for quite time about 11 hours after the end of SPE was $2.65 \mu \mathrm{Gy} / \mathrm{h}$. The additional absorbed dose at $40 \mathrm{~mm}$ depth in the phantom received from SPE was approximately $180 \mu \mathrm{Gy}$. Outside the SAA during the SPE the radiation quality factor was 2.5 leading to $21.1 \mu \mathrm{Sv} / \mathrm{h}$ dose equivalent rate at 40 $\mathrm{mm}$ depth in the phantom. After the SPE, the quality factor of GCR was 4.15 , leading to $11 \mu \mathrm{Sv} / \mathrm{h}$ dose equivalent rate outside the SAA. The additional dose equivalent at $40 \mathrm{~mm}$ depth in the phantom received from SPE was about $448 \mu \mathrm{Sv}$. The additional exposures received from SPE are comparable to the averaged daily absorbed dose and dose equivalent measured in the spherical phantom in ISS during quite periods. The accumulated doses during the SPE in Liulin-5 detectors, located in the phantom in MIM1 module of the ISS are lower, than the doses accumulated in the non-shielded detectors DB-8\#1 and DB$8 \# 4$ of the radiation monitoring system on the ISS, located in different positions in the service module of the ISS. There is a good agreement of Liulin- 5 dose rates trend during the SPE with the proton flux of energies $\geq 100 \mathrm{MeV}$ (able to penetrate inside ISS) measured by GOES -13 satellite.

\section{Acknowledgement}

This work is partly supported by grant DID-02/8 from the National Science Fund and by the Agreement between BAS and RAS on fundamental space research. Authors are thankful to RKK Energia and the cosmonauts for conducting the experiments with Liulin-5 on ISS. The authors are obliged to NIRS, ChibaJapan for the organization of the calibrations of Liulin-5 instrument at HIMAC.

\section{References}

1. Apathy I, Akatov YuA, Arkhangelsky VV, Bodnar L, Deme S, et al. (2007) TL measurements on board the Russian segment of the ISS by the "Pille" system during Expedition -8, -9 and -10 Acta Astronaut 60: 322-328.

2. Semkova J, Koleva R, Maltchev St, Bankov N, Benghin V, et al. (2012) Radiation characteristics in the spherical tissue-equivalent phantom on the ISS during solar activity minimum according the data from Liulin-5 experiment, Journal of Atmospheric and Solar-Terrestrial Physics 99: 157-163.

3. Golightly MJ, Hardy AC, Atwell W. Hardy K (1992) Description, analysis and impact of major solar activity during recent U.S. shuttle missions. Adv. Space Res. 12: 335-338.

4. Lobakov AP Liagushin VI, Panasiuk MI, Shavrin PI, Makhmutov VS, et al. (1992) Increase of solar cosmic rays on the "MIR" Space station in orbit during September-October 1989. Nuclear Tracks and Radiation Measurements 20: 59-64.

5. Shurshakov VA, Petrov VM, Panova NA, Ivanov YuV, Makhmutov VS et al.(1996) Experimental investigations of quasistable radiation belts formed after solar proton events in September-October 1989 and March 1991 based on measurements made by Liulin dosimeter-radiometer on board the Mir space station. Advances in Space Research 18: 251-256.

6. Dachev TsP, Semkova JV, Matviichuk YuN, Tomov BT, Koleva RT, et al.(1998) Inner Magnetosphere Variations after Solar Proton Events. Observations on MIR Space Station in 1989-1994 Time Period. Adv. Space Research 22: 521-526.

7. Dachev TsP, Matviichuk YuN, Semkova JV, Koleva RT, Boichev B, et al. (1989) Space radiation dosimetry with active detection's for the scientific program of the second Bulgarian cosmonaut on board the MIR space station. Adv. Space Res 9: 247-251.

8. Dachev TsP, Matviichuk YuN, Bankov NG, Semkova JV, Ivanov YaJ, et al.(1992) "Mir" Radiation Dosimetry Results during the Solar Flares Events in September-October 1989. Adv. Space Research 12: 321-324.

9. Smart DF, Shea MA, Dachev TsP, Bankov NG, Petrov VM, et al. (1994) The Dose Rate Observed on 19-21 October 1989 and its Modulation by Geophysical Effects. Adv. Space Res.14: 651-654.

10. Petrov VM, Machmutov VS, Panova NA, Shurshakov VA, Dachev TsP, et al. (1994) Peculiarities of the Solar Proton Events of 19October 1989 and 23 March 1991 according to the Measurements on board the MIR Space Station. Adv. Space Res. 14: 645-650.

11. Shurshakov VA, Petrov VM, Ivanov YuV, Bondarenko VA, Tzetlin VV, et a (1999) Solar particle events observed on MIR station. Radiation Measurements 30: 317-325.

12. Kartsev IS, Akatov YuA, Eremenko VG, Petrov VI, Petrov VM, et al. (2005) Spherical phantom for studying radiation conditions in outer space. Designstructural special features. Nuclear Measurement \& Information Technologies 16: $36-45$.

13. Semkova J, Koleva R, Maltchev S, Kanchev N, Benghin V, et al. (2010) Radiation measurements inside a human phantom aboard the International Space Station using Liulin-5 charged particle telescope. Adv. Space Res.45 858-865.

14. Semkova J, Koleva R (2010) Overview on the Radiation Quantities Observed by Liulin-5 Instrument in a Human Phantom on International Space Station during the Minimum of 23rd Solar Cycle. Compt. Rend. Acad. Bulg. Sci 63 1533-1542.

15. Semkova J, Koleva R, Maltchev St, Bankov N, Benghin V, et al.(2012) Depth dose measurements with the Liulin- 5 experiment inside the spherical phantom of the Matroshka-R project onboard the International Space Station. Advances in Space Research 49: 471-478.

16. ICRP Report No. 60 (1991) Pergamon Press, Oxford.

17. Benghin VV, Petrov VM, Kireeva SA, Markov AV, Volkov AN, et al. (2005) Analysis of radiation dose increase caused by solar cosmic ray events observed by the radiation monitoring system on the Russian segment of the International Space Station. Advances in Space Research 36: 1749-1752. 